\title{
El método lúdico para lograr competencias de resolución de problemas de cantidad en niños y niñas de cinco años en una Institución Educativa de Ucayali, Perú
}

\section{The ludic method to achieve quantity problem-solving competence in five-year-old boys and girls in an Educational Institution in Ucayali, Perú}

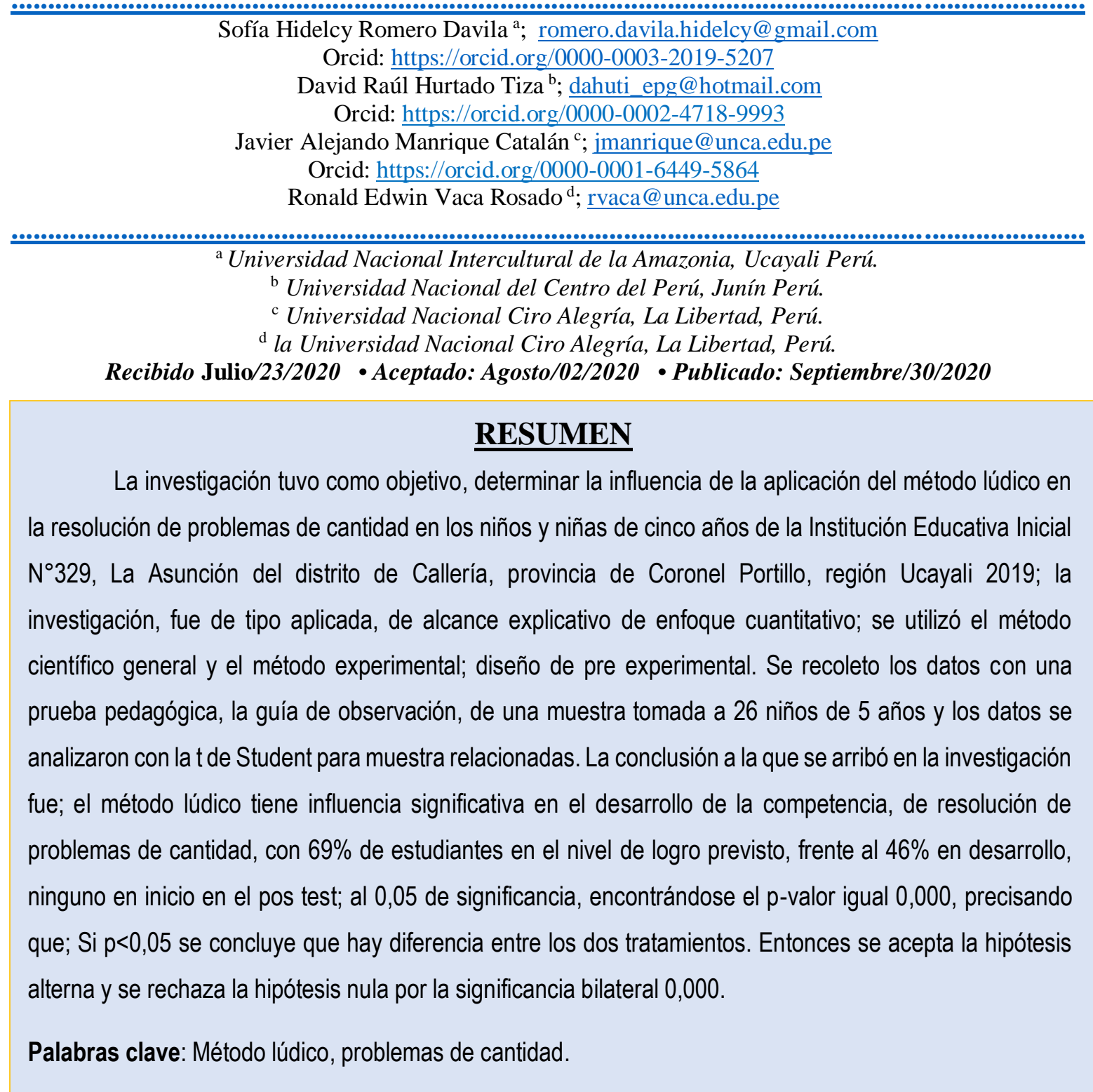

a . Sofía Hidelcy Romero - Davila; ${ }^{\mathrm{b}}$ David Raúl Hurtado - Tiza; ${ }^{\mathrm{c}}$ Javier Alejando Manrique - Catalán y ${ }^{\mathrm{d}}$ Ronald Edwin Vaca - Rosado

Articulo Protegido por Licencia Creative Commons: BY-NC-ND / Protected by Creative Commons: BY-NC-ND.

Sendas es una revista de acceso abierto / Sendas is an Open Access Journal. 


\section{ABSTRACT}

The objective of the research was to determine the influence of the application of the playful method in solving quantity problems in five-year-old boys and girls of the Initial Educational Institution No. 329, La Asunción, Callería district, Coronel Portillo province. , Ucayali region 2019; The investigation was of an applied type, of explanatory scope of quantitative approach; the general scientific method and the experimental method were used; pre-experimental design. The data was collected with a pedagogical test, the observation guide, from a sample taken from 265 -year-old children and the data was analyzed with the Student's $t$ test for related samples. The conclusion reached in the investigation was; The playful method has a significant influence on the development of competence, solving quantity problems, with $69 \%$ of students at the expected level of achievement, compared to $46 \%$ developing, none at the beginning in the post-test; at 0.05 of significance, finding the $p$-value equal to 0.000 , specifying that; If $p<0.05$ it is concluded that there is a difference between the two treatments. Then the alternate hypothesis is accepted and the null hypothesis is rejected by the bilateral significance 0.000 .

Keywords: Playful method, quantity problems.

\section{Introducción}

El aprendizaje de las matemáticas en niños de educación inicial es un tema de interés en la educación peruana, su éxito o deficiencia en esta competencia repercute en la sociedad por generaciones. La educación inicial es el primer peldaño de la generación de conocimiento, cumple un rol muy importante, ya que su impacto recae en el bienestar físico y motriz, en las habilidades del lenguaje, la comprensión de conceptos matemáticos, la autorregulación del propio aprendizaje y de las emociones.
Los resultados en educación en las últimas décadas no han sido muy alentadores para los países de Latinoamérica y el Caribe. En promedio, aproximadamente un tercio de los alumnos en primaria y casi la mitad en secundaria no parecen haber adquirido los aprendizajes básicos en lectura, y en matemáticas los resultados son incluso menos satisfactorios". UNESCO (2013), entendiéndose esto como una situación global que adolece desde la formación inicial.

El análisis de este organismo internacional hace el comentario reflexivo en

a Sofía Hidelcy Romero - Davila; ${ }^{\mathrm{b}}$ David Raúl Hurtado - Tiza; ${ }^{\mathrm{c}}$ Javier Alejando Manrique - Catalán y ${ }^{\mathrm{d}}$ Ronald Edwin Vaca - Rosado

Articulo Protegido por Licencia Creative Commons: BY-NC-ND / Protected by Creative Commons: BY-NC-ND.

Sendas es una revista de acceso abierto / Sendas is an Open Access Journal. 
tanto encuentra cierta debilidad en las políticas de estado, acerca de las condiciones, materiales y capacidad profesional entre otras. "Las políticas educacionales debieran enfocarse en asegurar en cada escuela los insumos, las condiciones organizacionales y las capacidades profesionales para generar mejores oportunidades de aprendizaje para todos los alumnos, especialmente los que enfrentan mayores dificultades".

En el caso peruano, la situación es parte de los casos citados por la UNESCO con respecto a los aprendizajes en los últimos años. La UNICEF (2015) hacía ya un anuncio en la medida que "Si bien Perú está cada vez más cerca de lograr la universalización de la educación primaria, existe el consenso de que asistir a la escuela no necesariamente se está traduciendo en que los niños y niñas tengan una trayectoria escolar exitosa, en la que incorporen los conocimientos y capacidades que por derecho les corresponde".

$$
\text { Según la UNICEF (2015), “el }
$$
problema educativo más grave que afecta a las niñas y niños del Perú es el bajo nivel de comprensión lectora y razonamiento matemático. Se trata de dos competencias básicas del proceso de aprendizaje sin las cuales las niñas y los niños peruanos verán limitados su desarrollo integral y sus oportunidades de llegar a la adultez como adultos productivos y ciudadanos plenos". El reporte sobre la prueba ECE, del Ministerio de Educación del Perú, MINEDU (2016), señala en años consecutivos del siguiente modo: en el 2015, el $31 \%$ se encentró en nivel de inicio; el $42.3 \%$ se encuentra en el nivel de proceso de sus aprendizajes; luego el $26 \%$ alcanza un nivel satisfactorio en aprendizaje de matemática. El año 2016, en el segundo grado de primaria en matemáticas, el $28 \%$ se encuentra en nivel de inicio; el $37.3 \%$ se encuentra en el nivel de proceso de aprendizaje; luego el $34.1 \%$ alcanza el nivel satisfactorio.

Se puede apreciar que el aprendizaje del área de matemática en la educación peruana aún sigue con bajos resultados, se considera de prioridad de primer orden superar esta dificultad. Los resultados de PISA 2012, fueron muy desalentadores, en donde el Perú ocupó el último lugar del ranking de 66 países, el Perú obtuvo 373 puntos en Ciencias, en comparación con el puntaje más alto obtenido por Chile que fue de 445, aunque este resultado es preocupante se le puede considerar como un reflejo de nuestro sistema educativo. Si bien es cierto que en la

a . Sofía Hidelcy Romero - Davila; ${ }^{\mathrm{b}}$ David Raúl Hurtado - Tiza; ${ }^{\mathrm{c}}$ Javier Alejando Manrique - Catalán y ${ }^{\mathrm{d}}$ Ronald Edwin Vaca - Rosado

Articulo Protegido por Licencia Creative Commons: BY-NC-ND / Protected by Creative Commons: BY-NC-ND.

Sendas es una revista de acceso abierto / Sendas is an Open Access Journal. 
evaluación PISA 2015 el Perú obtuvo alguna mejora, como por ejemplo en ciencias de 373 puntos se incrementó a 397 puntos, sin embargo, existen diversos aspectos por mejorar.

La realidad en la región Ucayali en la amazonia del Perú, la prueba ECE del año 2018 en la Región Ucayali, para el Área de Matemática reporta que en el nivel previo al inicio se encuentra el $25.5 \%$ y en el nivel inicio $31.6 \%$ en el nivel de proceso $32.1 \%$ y satisfactorio $10.1 \%$. Como quiera que, a diferencia del año 2016, hay una ligera mejora en todos los niveles, sin embargo, el porcentaje en los niveles de previo al inicio e inicio siguen siendo altas.

En cuanto al desempeño de los niños y niñas, en la I.E. N³29 "La Asunción" distrito de Callería, provincia de Coronel Portillo, se puede apreciar que los métodos y recursos aplicados en los procesos de enseñanza aprendizaje requieren de innovación. En algunos casos, hay cierta dureza de parte de los docentes con los niños, escasa variación, de métodos para aprendizajes útiles y significativos para su grupo social y su cultura, a partir de las nuevas tecnologías que vayan surgiendo. Se considera que los métodos lúdicos son los más indicados para lograr aprendizajes significativos, siempre en cuando se apliquen bajo un enfoque pedagógico unido al contexto.

Es innegable que el conocimiento matemático es una herramienta básica para la comprensión y manejo de la realidad en que vivimos. Su aprendizaje, además de durar toda la vida, debe comenzar lo antes posible para que el niño se familiarice con su lenguaje, su manera de razonar y de deducir. En la etapa de la Educación Inicial, el conocimiento se construye de manera global, y ésta disciplina no es una excepción. Cualquier situación puede aprovecharse para el desarrollo de los conceptos matemáticos. Piaget dice: “el aprendizaje es un proceso de adquisición de operaciones" Esto significa que los alumnos deberán convertirse en los protagonistas de un camino que iremos marcando con nuestras propuestas. Surge la interrogante ¿Cómo influye la aplicación del método lúdico en la resolución de problemas de cantidad en los niños y niñas de cinco años?

Revisando otros estudios de la variable, se encontró a Vales (2011) acerca: $E l$ juego como estrategia para la enseñanza de las matemáticas en la educación pre escolar. Encontraron que el trabajo desarrollado en las estrategias lúdicas para mejorar los

a . Sofía Hidelcy Romero - Davila; ${ }^{\mathrm{b}}$ David Raúl Hurtado - Tiza; ${ }^{\mathrm{c}}$ Javier Alejando Manrique - Catalán y ${ }^{\mathrm{d}}$ Ronald Edwin Vaca - Rosado

Articulo Protegido por Licencia Creative Commons: BY-NC-ND / Protected by Creative Commons: BY-NC-ND.

Sendas es una revista de acceso abierto / Sendas is an Open Access Journal. 
aprendizajes significativos en el área de matemática en los niños de 5 años de educación inicial de la I.E. Nro. 081 caserío Santa Isabel, distrito de Mórrope se observa que existe un $53 \%$ (C) de estudiantes que se encuentra en el nivel de inicio, el 93\% (A) ha alcanzado el logro previsto; es decir, ha influido la estrategia aplicada.

Por su parte, Ortegamo \& Bracamonte (2011) trabajaron, “Actividades lúdicas como estrategia didáctica para el mejoramiento de las competencias operacionales en la enseñanza aprendizaje de la matemática básica", se pudo comprobar que las actividades lúdicas lograron que los estudiantes mejorarán el rendimiento académico de las operaciones básicas y que, paulatinamente sus niveles fueron aumentaron al grado de afianzamiento del conocimiento, es decir, estos resultados de motivación se extendieron a otros grados $y$ niveles de enseñanza.

Asimismo, Huamán (2016) investigó: Aplicación de un programa de juegos lúdicos para mejorar el aprendizaje en el área de matemática en los niños de 5 años en la I.E $N^{o}$ 82318 de Calluan, distrito de Cahachi, provincia de Cajabamba-2015. En las cuales llego la concluir que: 1) El aprendizaje de los niños de la muestra se ha obtenido que el 61.5 $\%$ tienen un nivel de aprendizaje B (Proceso); el 30.85 obtuvieron C (Inicio) y un 7.75 obtuvieron A (Logro Previsto). 2. Mediante la aplicación del Programa de Juegos Lúdicos se demuestra que el aprendizaje de los niños ha mejorado, evidenciándose en el Post - Test, con un $100 \%$ en el nivel de Logro Previsto.

También, Rojas (2019), investigó: Juego lúdico matemático en el desarrollo de competencias y capacidades matemáticas en niños de 5 años de la I.E.I. $N^{a} 676$ San Martin De Porras- Amay- Huacho. Concluyó que si existe relación significativa entre el juego lúdico matemático y el desarrollo de competencias y capacidades matemáticas en niños de 5 años de la I.E.I. N ${ }^{a} 676$ San Martín de Porras - Amay, con una correlación de Spearman de 0.469, representando una moderada asociación." En efecto, el juego lúdico es importante en el desarrollo del niño, porque le permite el placer de hacer las cosas, de imaginarlas distintas a como se nos aparecen.

El juego lúdico, según Chamorro (2007, p.289) “el método lúdico es un conjunto de métodos que están destinados al logro de aprendizajes. Es una secuencialización de actividades que pretenden desarrollar

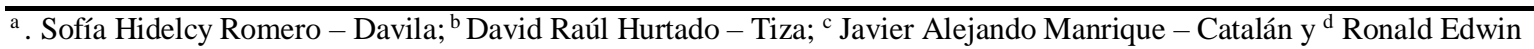
Vaca - Rosado

Articulo Protegido por Licencia Creative Commons: BY-NC-ND / Protected by Creative Commons: BY-NC-ND.

Sendas es una revista de acceso abierto / Sendas is an Open Access Journal. 
capacidades, teniendo ésta en su anterior métodos dirigidos o estructurados que han pasado por un proceso de contextualización y orientados a un determinado fin”. Según Motta, (1998) "La lúdica es un procedimiento pedagógico en sí mismo, que genera espacios y tiempos lúdicos, provoca interacciones y situaciones lúdicas, cuya práctica desarrolla habilidades de convivencia, a coexistir a partir de valores individuales y colectivos mediante el juego".

Para Dinello (1988:41) la ludoteca es: Un espacio lúdico, un mundo real transformado cada día por la imaginación de niños jugando. La ludoteca ofrece todo de juguetes, pero principalmente, es un ambiente de libertad lúdico creativa. "Las ludotecas son un espacio para favorecer la expresión de cada participante donde a través de actividades múltiples, tanto los niños como los adultos que los acompañan se divierten con toda espontaneidad, al tiempo que se descubren $y$ estructuran como personas, son un espacio de socialización relativa". Según (Sefchovich, 1996) El Juego: es una sesión de expresión corporal siempre debe incluir como objetivo "el placer del movimiento y un sentido lúdico del trabajo y del aprendizaje". Los métodos de grupo refuerzan estas actitudes y permiten la salida gradual de la implicación profunda con el material con que se ha trabajado.

Para (Smith 2006, p. 13) establece que el juego consiste en la orientación del sujeto hacia su propia conducta, más que en un tipo de conducta particular. Este control sobre la propia actividad, que se contrapone al ejercicio originado por los estímulos externos, necesidades, metas propias de los comportamientos no lúdicos, tiene mucho que ver con la distorsión de la realidad que supone el proceso de asimilación, tanto biológico como psicológico al lograr que el niño a través del juego pueda desarrollar sus habilidades cognitivas.

Sobre la clasificación de los juegos lúdicos Según, Piaget (1945 p.187) considera una secuencia común del desarrollo del juego de manera jerarquizada clasificándolo en: El Juego Motor o de Ejercicio: Está asociado al movimiento logrando experimentar con el propio cuerpo las sensaciones que genera en el niño como por ejemplo cuando realiza actividades de juego como saltar en un pie, saltar con la soga, subir y bajar gradas, lanzar

a . Sofía Hidelcy Romero - Davila; ${ }^{\mathrm{b}}$ David Raúl Hurtado - Tiza; ${ }^{\mathrm{c}}$ Javier Alejando Manrique - Catalán y ${ }^{\mathrm{d}}$ Ronald Edwin Vaca - Rosado

Articulo Protegido por Licencia Creative Commons: BY-NC-ND / Protected by Creative Commons: BY-NC-ND.

Sendas es una revista de acceso abierto / Sendas is an Open Access Journal. 
una pelota, columpiarse, correr, otros son métodos motores.

Por su parte, Ortega (1995), podemos considerar cinco tipos de métodos:

a) El Juego de Reglas: Se da entre los 7 y los 11 años. En esta fase la competición entra con más fuerza, pues las personas no alcanzan a disociar entre juego y competición creyendo que una no puede vivir sin la otra. Este tipo de juego se utiliza para la competición, pero también para los de cooperación (esperando su turno, respetando a su compañero). Hasta los 7 años las reglas son inflexibles y sagradas, después empieza hacer producto de acuerdos y modificadas por consenso. El juego de reglas también va ser parte de la vida del adulto, ya que es una actividad lúdica de un ser socializado. En resumen, el juego de reglas:

- Es aprendizaje de estrategias de interacción social.

- Facilita el control de la agresividad.

- Es ejercicio de responsabilidad y democracia

b) Métodos de construcción-representación. Son una forma evolucionada de los métodos sensorios motores ya que incluyen una simbolización sobre la acción que se realiza. Contiene una parte individual y otra que es externa y social. Tiene lugar mayoritariamente en el contexto familiar.

Podemos distinguir tres tipos: Métodos de representación incipiente: El niño comienza a utilizar el lenguaje como un elemento más del juego e incorpora progresivamente símbolos cada vez más variados y complejos. Métodos de representación vicaria: Los niños reproducen los roles sociales y desarrollan guiones de la actividad humana, haciendo, a veces, que sus personajes sean encarnados por pequeños muñecos o por animales de simulación. Pueden representar varios papeles, prestando sus voces a cada uno de los personajes. Sus elementos son: escenario, acción y personajes.

c) Métodos de contacto físico. Son métodos de carrera, persecución, ataque y dominación física. Tiene Su origen en el juego sensorio motor, pero incorpora muy pronto la presencia de un compañero del juego con el que interactúa imitando un supuesto ataque que se vive con alegría y entusiasmo. El componente mayor es de simulación y de contacto físico. Son frecuentes entre los tres y ocho años.

Los juegos se caracterizan, según (rutas de aprendizaje 2013 p.68) El juego es

a . Sofía Hidelcy Romero - Davila; ${ }^{\mathrm{b}}$ David Raúl Hurtado - Tiza; ${ }^{\mathrm{c}}$ Javier Alejando Manrique - Catalán y ${ }^{\mathrm{d}}$ Ronald Edwin Vaca - Rosado

Articulo Protegido por Licencia Creative Commons: BY-NC-ND / Protected by Creative Commons: BY-NC-ND.

Sendas es una revista de acceso abierto / Sendas is an Open Access Journal. 
ante todo divertido y placentero, aunque no se manifiesten signos de alegría, siempre es considerado positivamente por quien lo practica. Todo juego posee unas características generalmente aceptadas por todos los autores, por consiguiente, podrían ayudarnos a diferenciar el juego, de cualquier otra actividad.

Estos rasgos diferenciales son:

- Placentero

- Espontáneo

- Voluntario en su forma original

- Tiene un fin en sí mismo

- Es expresivo, comunicativo y explorador

- Implica cierta participación activa por arte de los jugadores

El rol del docente dentro de la estrategia de aprendizaje del juego debe estar bien delimitado, manteniéndose al margen ya que debe permitir que los niños se apropien mayormente de las actividades del juego es como guía, conjuntamente con el enfoque problematizado del campo formativo, en estas situaciones el rol puede ser también de facilitador.

Según Vargas (2009 p.89) establece que: "Nuestro rol como mediador en los métodos permite mezclarse en las situaciones de juego, de conflicto que pudieran registrar de los métodos sociales o de los métodos reglados, el adulto puede tomar un papel arbitrario en el desarrollo de los métodos en el que señale las actitudes que logran el buen desarrollo del juego en el proceso del aprendizaje".

Es así como Lezama (2016) en su estudio refiere a pesar de su actitud proactiva, los profesores experimentan una gran frustración al ver que los resultados de sus acciones no conducen a los resultados esperados (p.97). La ilusión por mejorar la situación del alumno no permite que el afecto sea notorio, sin embargo, el afecto brindado por el maestro es esencial para el estudiante, porque despierta en él emociones, sentimientos y/o pasiones que determinarán su formación.

Acerca de la resolución de problemas de cantidad de la competencia de las matemáticas para educación inicial, según el Currículo Nacional (2016), la competencia: Resuelve problemas referidos a relacionar objetos de su entorno según sus características perceptuales; agrupar, ordenar hasta el quinto lugar, seriar hasta 5 objetos, comparar cantidades de objetos y pesos, agregar y quitar hasta 5 elementos, realizando representaciones

a . Sofía Hidelcy Romero - Davila; ${ }^{\mathrm{b}}$ David Raúl Hurtado - Tiza; ${ }^{\mathrm{c}}$ Javier Alejando Manrique - Catalán y ${ }^{\mathrm{d}}$ Ronald Edwin Vaca - Rosado

Articulo Protegido por Licencia Creative Commons: BY-NC-ND / Protected by Creative Commons: BY-NC-ND.

Sendas es una revista de acceso abierto / Sendas is an Open Access Journal. 
con su cuerpo, material concreto o dibujos. Expresa la cantidad de hasta 10 objetos, usando estrategias como el conteo. Usa cuantificadores: "muchos" "pocos", "ninguno", y expresiones: "más que" "menos que". Expresa el peso de los objetos "pesa más", "pesa menos" y el tiempo con nociones temporales como "antes o después", "ayer" "hoy" o "mañana".

En la actualidad, la presencia de la información cuantitativa se ha incrementado de forma considerable. Este hecho exige al ciudadano construir modelos de situaciones en las que se manifiesta el sentido numérico y de magnitud, lo cual va de la mano con la comprensión del significado de las operaciones y la aplicación de diversas estrategias de cálculo y estimación.

Treffers (citado por Jan de Lange) citado por MIDEU (2015) hace hincapié en la importancia de la capacidad de manejar números y datos, y de evaluar los problemas y situaciones que implican procesos mentales y de estimación en contextos del mundo real.

Por su parte, The International Life Skills Survey (Policy Research Initiative Statistics Canada, 2000) citado por MIDEU (2015) menciona que es necesario poseer "un conjunto de habilidades, conocimientos, creencias, disposiciones, hábitos de la mente, comunicaciones, capacidades y habilidades para resolver problemas que las personas necesitan para participar eficazmente en situaciones cuantitativas que surgen en la vida y el trabajo".

En las Rutas de Aprendizaje (2015) considera que: Desarrollar esta competencia "Actuar y pensar en situaciones de cantidad" en el II ciclo, implica que los niños hagan matemática al resolver problemas aditivos simples con acciones de agregar o quitar, comunique sus ideas matemáticas con respecto al significado del número y las operaciones empleando lenguaje matemático, es decir desarrolle nociones básicas, como la clasificación, la seriación, la cardinalidad, la ordinalidad, la correspondencia, etc. usando expresiones como: muchos, pocos, ninguno o más que, menos que, etc. al comparar cantidades, use diferentes estrategias de conteo con cantidades hasta $10 \mathrm{y}$ razone y argumente explicando en su propio lenguaje sus razones de cómo agrupó, ordenó o resolvió el problema.

\section{Metodología}

a . Sofía Hidelcy Romero - Davila; ${ }^{\mathrm{b}}$ David Raúl Hurtado - Tiza; ${ }^{\mathrm{c}}$ Javier Alejando Manrique - Catalán y ${ }^{\mathrm{d}}$ Ronald Edwin Vaca - Rosado

Articulo Protegido por Licencia Creative Commons: BY-NC-ND / Protected by Creative Commons: BY-NC-ND.

Sendas es una revista de acceso abierto / Sendas is an Open Access Journal. 
Respetando los criterios expuestos por Mejía (2013) la investigación corresponde a la siguiente tipología. Según el carácter de la medida es cuantitativa, debido a que la recolección de datos y el tratamiento e interpretación fueron mediante datos paramétricos de intervalos con valores numéricos. Según la profundidad es explicativa, puesto que pretende explicar la causa y efecto entre variables; es decir la aplicación de la variable independiente, el método lúdico sobre la variable independiente situaciones de cantidad de la competencia del área de matemática en el nivel inicial.

Según el alcance temporal es longitudinal debido a que los instrumentos fueron aplicados en un más de una vez a la muestra determinada. Según la orientación que asume, está orientada a la explicación mediante la pre experimentación. Se trabajó con una muestra no probabilística de 26 niños de cinco años de ambos sexos. Los datos fueron analizados utilizando la estadística descriptiva inferencial; la prueba de hipótesis se hizo aplicando la t de Student para muestras relacionadas. El diseño viene a ser pre experimental; toda vez que se trabajó con un solo grupo de estudio con un tipo de muestreo por decisión del investigador al estar ya definida la población con quienes se desarrollaron las actividades, método lúdico para la mejora de los aprendizajes de la matemática en la competencia situaciones de cantidad.

\section{GE: 01 X 02}

Dónde:

GE : Es el grupo experimental

01 y 02 : Es la evaluación inicial y final

$\mathbf{X}$ : Es la variable experimental o de tratamiento.

La aplicación de la variable, juegos lúdicos se desarrolló en 15 sesiones activas con el grupo de la muestra, niños de 5 años, organizado dos sesiones por semana. Los criterios para las sesiones fue la operacionalización de la variable donde cada dimensión tuvo actividades y estas se convirtieron en sesiones de 45 minutos cada una, ejemplo: Sesión 1: ...aprendemos agregar Sesión 2: ....aprender a quitar Sesión 3: ...aprendemos agrupar Sesión 4: ...aprendemos a contar Sesión 5: ...contamos hasta el 10...; asi hasta la sesión 15.

Se hizo dos mediciones, la prueba de entrada antes de iniciar con las actividades de aplicación o desarrollo de la variable independiente, luego se hizo la prueba de

a . Sofía Hidelcy Romero - Davila; ${ }^{\mathrm{b}}$ David Raúl Hurtado - Tiza; ${ }^{\mathrm{c}}$ Javier Alejando Manrique - Catalán y ${ }^{\mathrm{d}}$ Ronald Edwin Vaca - Rosado

Articulo Protegido por Licencia Creative Commons: BY-NC-ND / Protected by Creative Commons: BY-NC-ND.

Sendas es una revista de acceso abierto / Sendas is an Open Access Journal. 
salida conocida como pos test. Estos datos fueron sometidos al análisis y prueba de hipótesis.

\section{$\underline{\text { Resultados }}$}

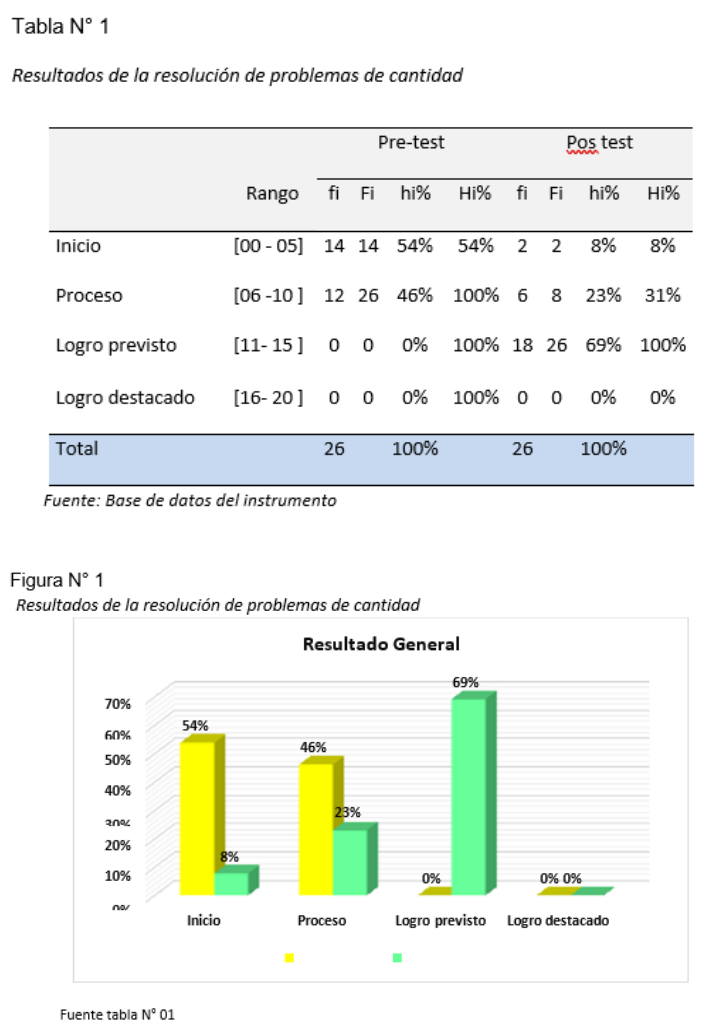

En la tabla y figura $\mathrm{N}^{\circ} 1$, se demuestra los resultados generales de pre test y pos test, sobre la variable dependiente resuelve problemas de cantidades; detallando que en el pre test de 26 niños y niñas, se ubicaron en el nivel inicio (14), 54\%, mientras en el nivel proceso, (12), $46 \%$, ninguno en el nivel logo previsto (0), $0 \%$ así mismo en el nivel logro destacado ninguno
(0), 0\%, estos resultados demuestran literalmente que existe la necesidad de mejorar y aplicar los métodos lúdicos después de desarrollar las sesiones prevista, en consecuencia tenemos el pos test los resultados muestran que se ubican en el nivel inicio (2), $8 \%$ niños y niñas, en el nivel proceso (6), 23\%, mientras en el logro previsto (18), $69 \%$ y en el logro destacado (0), estos resultados evidencian que implica la aplicación de los métodos lúdicos le permiten a los niños y niñas a resolver problemas relacionados con cantidades de manera eficiente donde que se pueden contar y medir para desarrollar progresivamente el sentido numérico y de magnitud, la construcción del significado de las operaciones, así como la aplicación de diversas estrategias de cálculo y estimación.

De los resultados de la prueba t de Student como todos los estadísticos de contraste se basa en el cálculo de estadísticos descriptivos previos: el número de observaciones, la media. -7,69231 y la desviación estándar, es 2,01533, se calcula el estadístico de contraste experimental. T. $-19,462$ con la ayuda de unas tablas se obtiene a partir de dicho estadístico el p-valor es 0.000, precisando que; Si p $<0,05$ se concluye que hay diferencia entre los dos

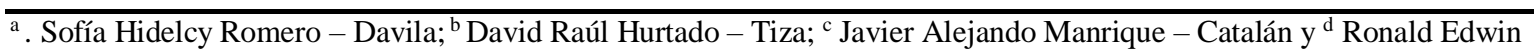
Vaca - Rosado

Articulo Protegido por Licencia Creative Commons: BY-NC-ND / Protected by Creative Commons: BY-NC-ND.

Sendas es una revista de acceso abierto / Sendas is an Open Access Journal. 
tratamientos. Entonces se acepta la hipótesis alterna y se rechaza la hipótesis nula por la significancia bilateral 0,000.

\section{Discusión}

La investigación se propuso; determinar la influencia de la aplicación del método lúdico en la competencia de Resolución de Problemas de cantidad en los niños y niñas de cinco años de la Institución Educativa N³29 La Asunción, del Distrito de Callería Coronel Portillo Ucayali 2019. Los resultados obtenidos demuestran que, en la prueba del pos test, el 69\% de los niños y niñas de cinco años alcanzó un nivel de logro previsto, lo que en el pre test no hubo. Solo se el $46 \%$ alcanzó el nivel proceso y luego el 5\% se ubicaba en inicio.

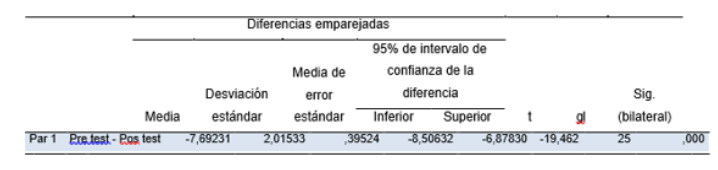

El estudio tiene coherencia con sus resultados de Zeña (2018) su trabajo de investigación fue: Estrategias lúdicas para mejorar los aprendizajes significativos en el área de matemática en los niños de cinco años de edad de educación inicial de la I.E. $N^{a}$ 081 caserío Santa Isabel, distrito de Mórrope, provincia de Lambayeque- 2015. Llegando a la conclusión que; el desarrollo de las estrategias lúdicas para mejorar los aprendizajes significativos en el área de matemática en los niños de 5 años de educación inicial de la I.E. Nro. 081 caserío Santa Isabel, distrito de Mórrope, se observa que existe un $53 \%$ (C) de estudiantes que se encuentra en el nivel de inicio, el 93\% (A) ha alcanzado el logro previsto.

Según MINEDU, (2009, p. 64) "Estimula la comunicación y la cooperación con los iguales. Prepara al niño para su desenvolvimiento futuro en el mundo del trabajo. Estimula el desarrollo moral, ya que es escuela de autodominio, voluntad y asimilación de reglas de conducta. Facilita el autoconocimiento, el desarrollo de la conciencia personal. Ayuda en los procesos de adaptación socio- emocional.

\section{Conclusiones}

- Se demuestra que, si es, significativo la aplicación del método lúdico para la competencia de resolución de problemas de cantidad de la matemática de acuerdo a los resultados, corroborados, el p- valor

a . Sofía Hidelcy Romero - Davila; ${ }^{\mathrm{b}}$ David Raúl Hurtado - Tiza; ${ }^{\mathrm{c}}$ Javier Alejando Manrique - Catalán y ${ }^{\mathrm{d}}$ Ronald Edwin Vaca - Rosado

Articulo Protegido por Licencia Creative Commons: BY-NC-ND / Protected by Creative Commons: BY-NC-ND.

Sendas es una revista de acceso abierto / Sendas is an Open Access Journal. 
es 0.000 , precisando que; $\mathrm{Si} \mathrm{p}<0,05$ se concluye que hay diferencia entre los dos tratamientos.

- Se corrobora que es significativo, método lúdico en la traducción de cantidades a expresiones numéricas en los niños y niñas de cinco años en la muestra, entonces se demuestra con la prueba de hipótesis el p-valor es 0.000 , precisando que; $\mathrm{Si}$ p $<0,05$ se concluye que hay diferencia entre los dos tratamientos. Entonces se acepta la hipótesis alterna y se rechaza la hipótesis nula por la significancia bilateral 0,000.

- Se corrobora que si existe significancia en la aplicación del método lúdico en la comunicación y comprensión sobre los números y las operaciones en los niños y niñas de cinco años con los resultados demostradas en la prueba de hipótesis; el p-valor es 0.000 , precisando que; $\mathrm{Si} \quad \mathrm{p}<0,05$ se concluye que hay diferencia entre los dos tratamientos.

- Se corrobora que hay significancia de la aplicación del método lúdico en el https://doi.org/10.47192/rcs.v1i3.45

uso de estrategias y procedimientos de estimación y cálculo en los niños y niñas de cinco años, pues se demostraron en la prueba de hipótesis; el p-valor es 0.000, precisando que; $\mathrm{Si}$ $\mathrm{p}<0,05$ se concluye que hay diferencia entre los dos tratamientos.

\section{$\underline{\text { Referencias }}$}

Chamorro, M. C. (1992) El aprendizaje Significativo en Matemáticas, Alhambra Longman, Madrid.

Huamán R. (2016) Aplicación de un programa de juegos lúdicos para mejorar el aprendizaje en el área de matemática en los niños de 5 años en la I.E $N^{o} 82318$ de Calluan, distrito de Cahachi, provincia de Cajabamba 2015. Tesis sustentada en Universidad Los Ángeles de Chimbote Trujillo.

Jaramillo C. (2012), "El juego en los sectores para desarrollar capacidades matemáticas en niños de 4 años de una institución educativa del Callao."

Mejía E. (2013) Metodología de la Investigación. $3^{\circ}$ Edición. Editorial

a . Sofía Hidelcy Romero - Davila; ${ }^{\mathrm{b}}$ David Raúl Hurtado - Tiza; ${ }^{\mathrm{c}}$ Javier Alejando Manrique - Catalán y ${ }^{\mathrm{d}}$ Ronald Edwin Vaca - Rosado

Articulo Protegido por Licencia Creative Commons: BY-NC-ND / Protected by Creative Commons: BY-NC-ND.

Sendas es una revista de acceso abierto / Sendas is an Open Access Journal. 
2020.

URL: https://www.revistas.infoc.edu.pe/index.php/sendas

Email: revistasendas@infoc.edu.pe

de la Universidad Nacional Mayor de

San Marcos. Lima.

MINEDU (2013) Manual de Robótica Educativa en el Aula. $1^{\circ}$ Edición. Edit.

Ministerio de Educación del Salvador.

MINEDU (2009) Diseño Curricular Nacional.

http://www.minedu.gob.pe/DeInteres/ xtras/den_2009.pdf

Ministerio de Educación del Perú. (2015 p.

27) Rutas de Aprendizaje

Ministerio de Educación del Perú (2013).

Rutas de Aprendizaje

Montessori, M. (1912) El método de la Pedagogía Científica. Barcelona: Casa Editorial Araluce.

Motta, J. (1998). La lúdica, procedimiento pedagógico. Bogotá: Universidad Nacional.

Ortega, R. (1995). Jugar y aprender. Sevilla: DIADA.

Ortegamo, R. \& Bracamonte, M. (2011). Actividades lúdicas como estrategia didáctica para el mejoramiento de las competencias operacionales en la enseñanza aprendizaje de las
Vol. 1, N³, pp. 1-15 Septiembre 2020 ISSN $2708-6380$ https://doi.org/10.47192/rcs.v1i3.45

matemáticas básicas. Tesis de posgrado. Universidad Andina.

Piaget, J. (1980). La formación del símbolo en el niño. México: fce.

Piaget, J. y B. Inhelder (1956). La psicología del niño. . 1a edición. Madrid: Ed. Morata.

Smith, B. y J. M. Roberts (2006). "Play, Games and Sport". En: H. C. Triandis y A. Herron: Handbook of Cross-Cultural Psychology, vol. 4. Boston: Allyn \& Bacon, Inc. Faustino Sánchez Carrión.

Rojas K. (2019) Juego lúdico matemático en el desarrollo de competencias y capacidades matemáticas en niños de 5 años de la I.E.I. $N^{a} 676$ San Martin de Porras- Amay - Huacho Tesis sustentada en la Universidad Faustino Sánchez Carrión.

Sefchovich, G. y Waisburd, G. (2005). Expresión corporal y creatividad. Sevilla: MAD.

Sefchovich (1996) El Juego Expresión corporal y creatividad. México Trillas.

a . Sofía Hidelcy Romero - Davila; ${ }^{\mathrm{b}}$ David Raúl Hurtado - Tiza; ${ }^{\mathrm{c}}$ Javier Alejando Manrique - Catalán y ${ }^{\mathrm{d}}$ Ronald Edwin Vaca - Rosado

Articulo Protegido por Licencia Creative Commons: BY-NC-ND / Protected by Creative Commons: BY-NC-ND.

Sendas es una revista de acceso abierto / Sendas is an Open Access Journal. 
UNESCO (2013) Situación Educativa de América Latina y el Caribe: Hacia la educación de calidad para todos al 2015. Oficina Regional de Educación para América Latina y el Caribe (OREALC/UNESCO Santiago)

UNICEF (2015) Situación del país, educación primaria. Consultado el 11/12/17. Disponible en: https://www.unicef.org/peru/spanish /children_3787.html

Vargas, R. (2009). El cuento motor y su incidencia en la educación por el movimiento. Pensamiento educativo 38, 108-124.
Vales (2011) El juego como estrategia para la enseñanza de las matemáticas en la educación pre escolar. I.E. Nro. 081 caserío Santa Isabel, distrito de Mórrope.

Vygotsky, L. S. (1982). El papel del juego en el desarrollo. Barcelona: Crítica.

Zeña (2018) Estrategias lúdicas para mejorar los aprendizajes significativos en el área de matemática en los niños de cinco años de edad de educación inicial de la I.E. $N^{a} 081$ caserío Santa Isabel, distrito de Mórrope, provincia de Lambayeque- 2015.

El método lúdico para lograr competencias de resolución de problemas de cantidad en niños y niñas de cinco años en una Institución Educativa de Ucayali, Perú (Sofía Hidelcy Romero - Davila) Por Revista Sendas se encuentra bajo una Licencia Creative Commons-No Comercial-Sin Derivadas 3.0 Uported.

a . Sofía Hidelcy Romero - Davila; ${ }^{\mathrm{b}}$ David Raúl Hurtado - Tiza; ${ }^{\mathrm{c}}$ Javier Alejando Manrique - Catalán y ${ }^{\mathrm{d}}$ Ronald Edwin Vaca - Rosado

Articulo Protegido por Licencia Creative Commons: BY-NC-ND / Protected by Creative Commons: BY-NC-ND.

Sendas es una revista de acceso abierto / Sendas is an Open Access Journal. 